\title{
The Conserved 12-Amino Acid Stretch in the Inter-Bromodomain Region of BET Family Proteins Functions as a Nuclear Localization Signal
}

\author{
Hidesuke Fukazawa $^{*, a}$ and Atsuko Masumi ${ }^{b}$ \\ ${ }^{a}$ Department of Bioactive Molecules, National Institute of Infectious Diseases; 1-23-1 Toyama, Shinjuku-ku, Tokyo \\ 162-8640, Japan: and ${ }^{b}$ Department of Blood and Biological Products, National Institute of Infectious Diseases; \\ 4-7-1 Gakuen Musashimurayama, Tokyo 208-0111, Japan. \\ Received June 14, 2012; accepted September 2, 2012; advance publication released online September 7, 2012
}

The bromodomain and extraterminal (BET) family is a group of chromatin-binding proteins characterized by two bromodomains, an extraterminal (ET) domain, and several other conserved regions of unknown function. In humans, the BET family consists of four members, BRD2, BRD3, BRD4 and BRDT, that all normally localize to the nucleus. We identified a 12 -amino acid stretch in the inter-bromodomain region that is perfectly conserved among the BET family members. We deleted these residues and expressed the mutant proteins in HEK293T cells to investigate the function of this motif. We found that the deletion of this motif alters the localization of BET proteins. Mutated BRD3 and BRD4 were excluded from the nucleus, and BRDT was found to be diffused throughout the nucleus and cytoplasm. Although the mutant BRD2 remained predominantly in the nucleus, a punctate distribution was also observed in the cytosol. It has been reported that a conserved motif between the second bromodomain and the ET domain serves as a nuclear localization signal for BRD2. Nevertheless, BET mutants lacking the reported nuclear localization signal motif but retaining the 12-amino acid stretch resided in the nucleus. Furthermore, these mutants were diffused throughout the cytoplasm when the 12 residues were removed. These results indicate that the conserved amino acid stretch in the inter-bromodomain region of the BET family functions as a nuclear localization signal.

Key words bromodomain; BET family; epigenetics

Bromodomains are protein modules of approximately 110 amino acids that interact with acetylated lysine residues and play vital roles in the reading of epigenetic marks on histone tails. ${ }^{1)}$ The bromodomain and extraterminal (BET) family is a subgroup of bromodomain-containing proteins featuring two $\mathrm{N}$-terminal bromodomains and a C-terminal ET domain (Fig.
1). The human BET family of nuclear proteins consists of four members (BRD2, BRD3, BRD4 and BRDT) that have been implicated in various biological processes.

BRD4 binds directly to positive transcription elongation factor $b(p-T E F b)$, a complex of cyclin-dependent kinase (CDK) 9 and cyclin $\mathrm{T}$ that phosphorylates the RNA

\begin{tabular}{|c|c|c|c|c|c|}
\hline \multicolumn{6}{|c|}{182} \\
\hline BRD2 & BDI & BDII & & B & ET \\
\hline BRD2/BDI-BDII & BDI & BDII & & & \\
\hline
\end{tabular}

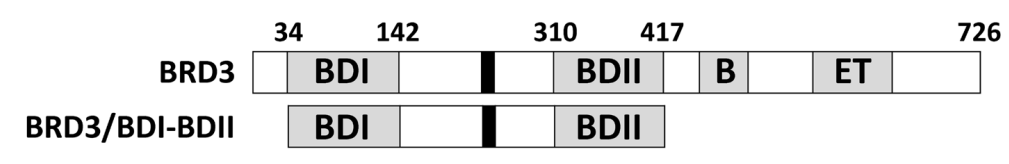

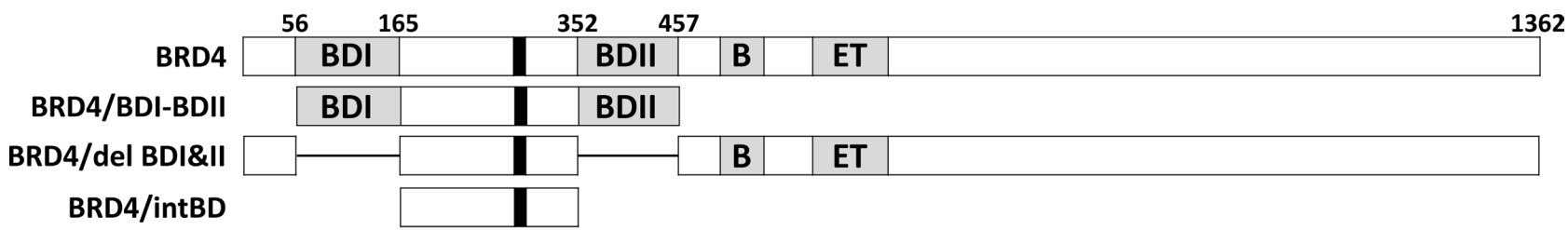

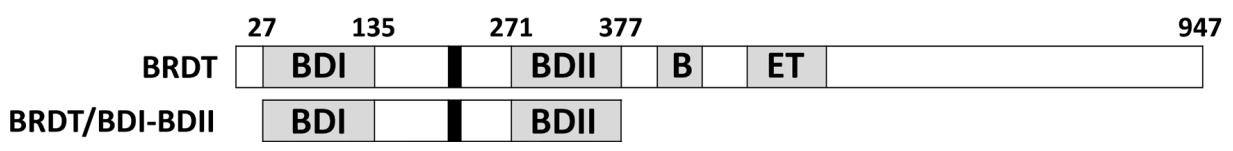

Fig. 1. Diagram of the BET Proteins and Deletions

BDI and BDII signify bromodomains. B and ET represent motif B and the ET domain, respectively. The black box indicates the conserved 12-amino acid stretch. BDI-BDII mutants span the two bromodomains and the sequences in between and lack the other regions. BRD4/del BDI\&II is a construct with deletions of both bromodomains. BRD4/intBD encodes a 188-amino acid segment between the two bromodomains.

The authors declare no conflict of interest.

* To whom correspondence should be addressed. e-mail: fukazawa@nih.go.jp 




Fig. 2. Deletion of the Conserved 12-Amino Acid Stretch in the Inter-Bromodomain Alters the Localization of BET Proteins

HEK293T cells were transfected with plasmids encoding the full-length (FL) or 12-amino-acid-deleted (del 12aa) EmGFP-BETs. The localization of EmGFP and DNA signals was examined using fluorescence microscopy $24 \mathrm{~h}$ after transfection.

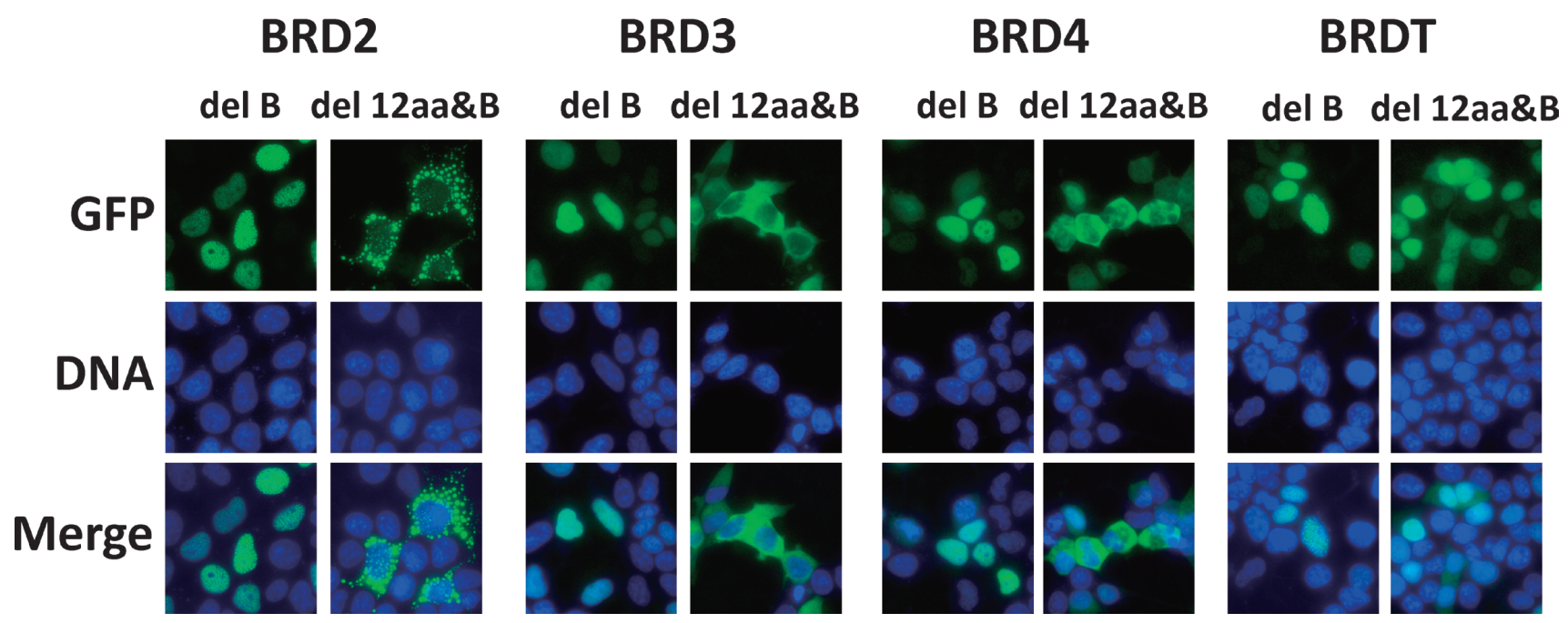

Fig. 3. Effects of Deletion of the Motif B Nuclear Localization Signal on Subcellular Localization of BET Proteins

HEK293T cells were transfected with plasmids encoding EmGFP-BETs that lack the motif B nuclear localization signal (del B) or both the inter-bromodomain 12-amino acid stretch and the motif B nuclear localization signal (del 12aa, B). The localization of EmGFP and DNA signals was examined as in Fig. 2.

polymerase II (RNAPII) C-terminal domain (CTD). BRD4 recruits $\mathrm{p}$-TEFb to acetylated chromatin, leading to increased RNAPII CTD phosphorylation and the stimulation of RNAPII-dependent transcription. ${ }^{2)}$ BRD2 and BRD3 also link histone acetylation and transcriptional regulation. ${ }^{3)}$

Abnormalities in BET protein functions can result in serious consequences, exemplified by chromosomal translocations in which the $\mathrm{N}$-terminal bromodomains of BRD4 and BRD3 are fused to the NUT (nuclear protein in testis) gene. ${ }^{4,5}$ Although the mechanism remains elusive, the formation of BRD4-NUT and BRD3-NUT fusion proteins results in a rare but lethal and aggressive form of squamous cell carcinoma known as NUT midline carcinoma (NMC). Other processes that have been demonstrated to involve BET proteins include DNA replication, ${ }^{6}$ ) the cell cycle, cancer metastasis, ${ }^{7-9)}$ the transmission of epigenetic memory, ${ }^{10)}$ chromatin remodeling, ${ }^{11}$ adipogenesis, ${ }^{12)}$ and viral genome segregation. ${ }^{13)}$ Another noteworthy characteristic of the BET proteins is their persistent association with chromatin throughout the cell cycle, including mitosis. ${ }^{13-16)}$ These structural and functional features of BET proteins suggest the importance of their nuclear localization; however, the mechanisms of the nuclear retention of BET proteins have not been thoroughly investigated.

In addition to the bromodomains and the ET domain, BET proteins share several other conserved regions of unknown function. We identified a 12-amino acid stretch (KGVKRKADTTTP) in the inter-bromodomain region that is perfectly conserved among the BET family of proteins (Fig. 1). To investigate the function of this motif, we deleted the 12 residues and expressed the mutant proteins tagged with GFP in HEK293T cells. Here, we demonstrated that this amino acid stretch plays a role in the nuclear retention of BET family proteins. 


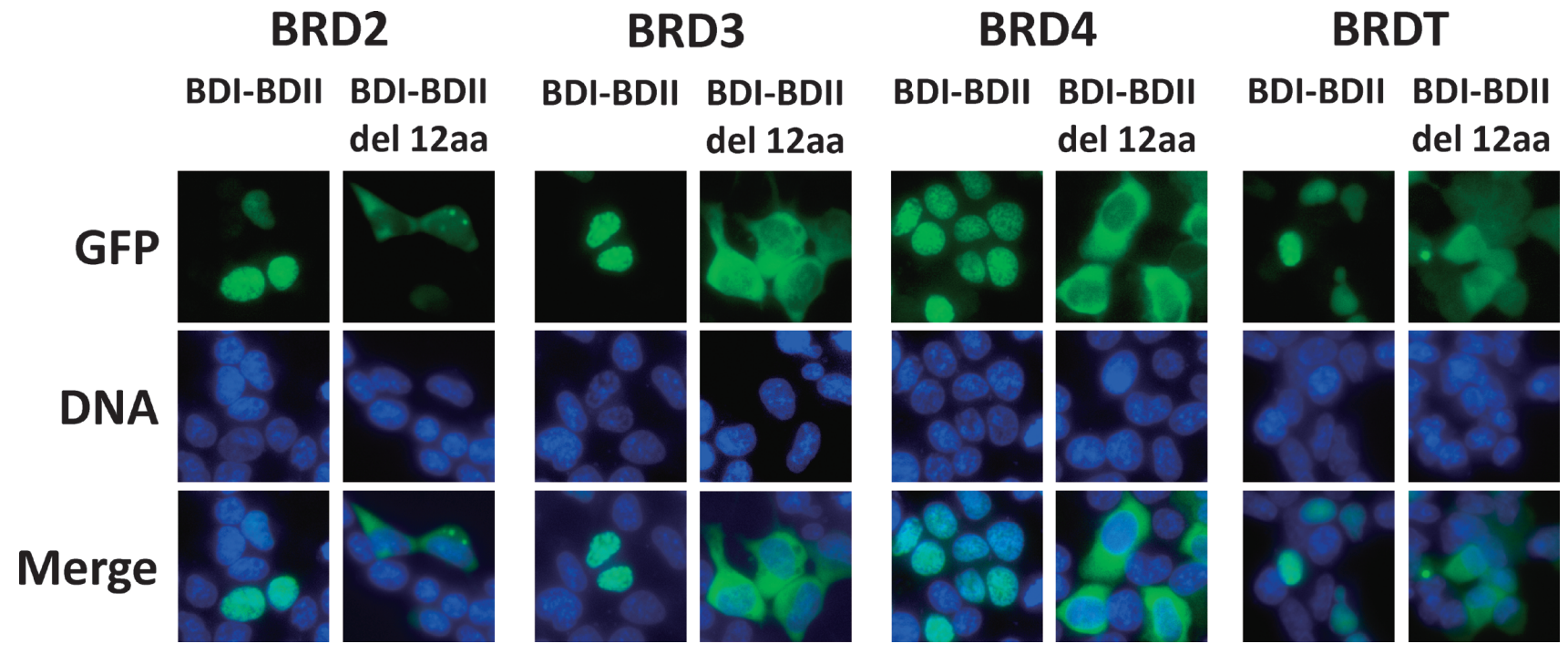

Fig. 4. BET Family BDI-BDII Mutants Are Localized to the Nucleus But Are Diffused throughout the Cytoplasm when the Conserved 12-Amino Acid Stretch Is Deleted

The HEK293T cells were transfected with the expression plasmids and examined as in Fig. 2.

\section{MATERIALS AND METHODS}

The human BET family cDNAs were isolated by PCR from the Human Mosaic cDNA Template (GENOFi, LLC, San Diego, CA, U.S.A.) and cloned into the pDONR221 entry vector (Invitrogen, Grand Island, NY, U.S.A.). The deletions were generated in Gateway entry clones using standard recombinant DNA technology. The genes were cloned into the pcDNA6.2/N-EmGFP-DEST vector to generate plasmids encoding the N-terminally emerald green fluorescent protein (EmGFP)-tagged BET proteins. HEK293T cells were seeded in 24-well plates at a density of $10^{5}$ cells per well and transfected with $400 \mathrm{ng}$ of the plasmids using TransIT 293 reagent. After 24h, the cells were stained with Hoechst 33342 and viewed using a Keyence BZ-9000 fluorescence microscope (Osaka, Japan).

\section{RESULTS AND DISCUSSION}

In addition to the two bromodomains and the ET domain, the BET family proteins share several other conserved motifs of poorly defined function (Fig. 1). ${ }^{17)}$ We observed a 12-amino acid stretch (KGVKRKADTTTP) in the inter-bromodomain that is perfectly conserved among the BET family proteins. This amino stretch is part of motif A, as identified by sequence alignments. ${ }^{18)}$ To assess the biological significance of this sequence, it was deleted from the sequences of the four members of the human BET family (BRD2, BRD3, BRD4 and BRDT), and the mutants were expressed as EmGFP fusions in HEK293T cells.

As shown in Fig. 2, the removal of these 12 amino acids altered the cellular distribution of the BET proteins. Whereas all of the full-length proteins localized to the nucleus, the BRD3 and BRD4 mutants devoid of the 12 residues were predominantly cytosolic, and truncated BRDT was diffused throughout the cytosol and nucleus. Although the BRD2 mutant remained predominantly nuclear, punctate signals were also detected in the cytosol. Our data are in accordance

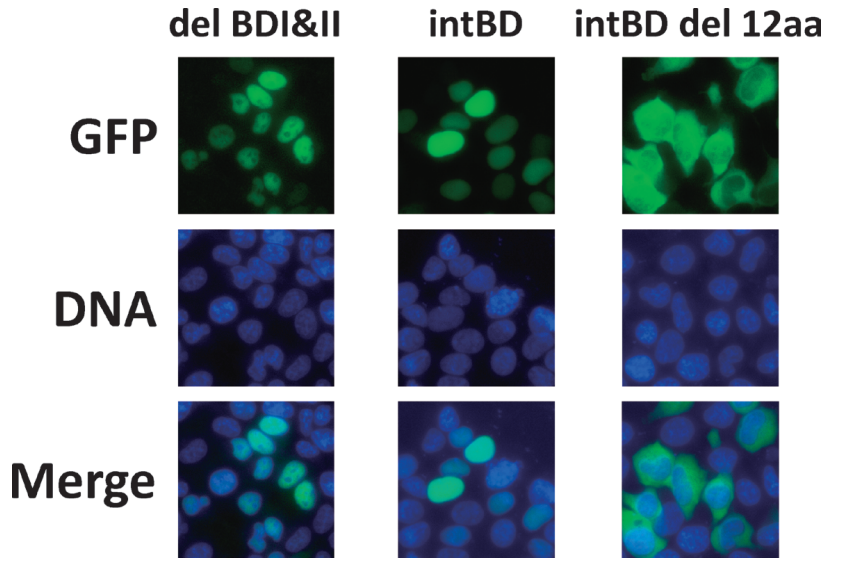

Fig. 5. Bromodomains Are Not Necessary for the Nuclear Localization of BRD4

HEK293T cells were transfected with plasmids encoding the double bromodomain deletion (del BDI\&II), the 188-amino acid inter-bromodomain segment (intBD), or the 12-amino-acid-deleted inter-bromodomain segment (intBD del 12aa) of BRD4 fused to EmGFP and the localization of EmGFP and DNA signals was examined.

with a previous report that the region separating the two bromodomains is at least partly responsible for the nuclear localization of mouse Brd4, ${ }^{6}$ ) and suggest the importance of the inter-bromodomain 12-amino acid motif in the subcellular localization of the BET proteins.

In contrast to the results for mouse Brd4, it has been reported that a classical nuclear localization sequence in BRD2, which is located between the second bromodomain and the ET domain (amino acids 554-559, KKKKRK), mediates the normal nuclear retention of BRD2. ${ }^{19)}$ This sequence is at the C-terminus of the domain referred to as motif $\mathrm{B}^{18)}$ Comparable sequences exist in other BET family members and have been predicted to function as nuclear localization sequences. ${ }^{4,12,20)}$

We deleted the amino acids containing the motif $\mathrm{B}$ nuclear localization signal from the BET proteins (amino acids 544-559 of BRD2, 487-504 of BRD3, 535-450 of BRD4 
and 447-463 of BRDT) and examined the localization of the mutants. Removal of these amino acids did not appear to have any influence on the nuclear retention of the BET proteins (Fig. 3). When the inter-bromodomain 12-amino acid stretch and the motif B nuclear localization signal were both deleted, BRD2 was mainly detected in the cytosol as dotted signals (Fig. 3). However, a substantial portion of BRDT still remained nuclear. As expected, the double deletion mutants of BRD3 and BRD4 were mainly cytosolic.

To address further the contribution of the conserved 12 amino acids to the subcellular localization of the BET proteins, we deleted the amino acids $\mathrm{N}$-terminal to the first bromodomain and those $\mathrm{C}$-terminal to the second bromodomain from the BET proteins. The resulting segments (BRD2/ BDI-BDII, BRD3/BDI-BDII, BRD4/BDI-BDII and BRDT/ BDI-BDII in Fig. 1) span the two bromodomains and thus contain the inter-bromodomain 12-amino acid stretch but lack other conserved regions including motif B. As shown in Fig. 4, all the BDI-BDII mutants were confined to the nucleus. Furthermore, these variants were diffused throughout the cytosol when the 12-residue motif in the inter-bromodomain was removed.

We also observed that the deletion of either bromodomain or both bromodomains of BRD4 (BRD4/del BDI\&II in Fig. 1) did not apparently alter the nuclear retention of this protein (Fig. 5 and not shown). Moreover, the inter-bromodomain region of BRD4 (BRD4/intBD in Fig. 1) alone localized to the nucleus, and was rendered mainly cytosolic by deletion of the 12-amino acid stretch (Fig. 5). However, unlike the full-length protein or other mutants that localized to the nucleus, BRD4/ intBD was evenly dispersed throughout the nucleoplasm and did not display any particular distribution within the nucleus, implying that other regions are required for precise subnuclear localization of BRD4. These results suggest that the nuclear compartmentalization of BET proteins is not mediated by the interaction of the bromodomains with chromatin.

The 12-amino acid stretch in the inter-bromodomain is present in vertebrate BET proteins and Drosophila Fsh but not in Saccharomyces cerevisiae Bdf1 and Bdf2. Yet, motif B is evolutionary conserved from yeast to humans. The inter-bromodomain motif may have evolved as a requirement for more efficient control of subcellular localization. Our data indicate that the 12-amino acid stretch in the inter-bromodomain is the major, if not the sole determinant of nuclear retention for BRD3 and BRD4, and is also important for the nuclear localization of BRD2 and BRDT. On the other hand, although motif B did appear to contribute to the localization of BRD2, we could not obtain any evidence for its involvement in the nuclear localization of BRD3, BRD4 or BRDT. It has recently been reported that the motif $\mathrm{B}$ is required for association to chromosomes and for homo- and hetero-dimerization between BET proteins. ${ }^{21)}$ The main function of motif $\mathrm{B}$ may not be nuclear retention. It is also possible that there are other domains that serve as a nuclear localization signal or a nuclear export signal.

In summary, we conclude that the conserved 12-amino acid sequence in the inter-bromodomain is at least part of the nuclear localization signal and plays important roles in the cytoplasmic/nuclear partitioning of the BET proteins.

Acknowledgments This study was supported by a
Grant-in-Aid from the Ministry of Health, Labour and Welfare of Japan, and a Grant-in-Aid for Scientific Research on Innovative Areas, Scientific Support Programs for Cancer Research, from the Ministry of Education, Culture, Sports, Science and Technology of Japan.

\section{REFERENCES}

1) Filippakopoulos P, Picaud S, Mangos M, Keates T, Lambert JP, Barsyte-Lovejoy D, Felletar I, Volkmer R, Müller S, Pawson T, Gingras $\mathrm{AC}$, Arrowsmith $\mathrm{CH}$, Knapp S. Histone recognition and large-scale structural analysis of the human bromodomain family. Cell, $\mathbf{1 4 9}$, 214-231 (2012).

2) Jang MK, Mochizuki K, Zhou M, Jeong HS, Brady JN, Ozato K. The bromodomain protein Brd4 is a positive regulatory component of P-TEFb and stimulates RNA polymerase II-dependent transcription. Mol. Cell, 19, 523-534 (2005).

3) LeRoy G, Rickards B, Flint SJ. The double bromodomain proteins $\mathrm{Brd} 2$ and Brd3 couple histone acetylation to transcription. Mol. Cell, 30, 51-60 (2008).

4) French CA, Miyoshi I, Kubonishi I, Grier HE, Perez-Atayde AR, Fletcher JA. BRD4-NUT fusion oncogene: a novel mechanism in aggressive carcinoma. Cancer Res., 63, 304-307 (2003).

5) French CA, Ramirez CL, Kolmakova J, Hickman TT, Cameron MJ, Thyne ME, Kutok JL, Toretsky JA, Tadavarthy AK, Kees UR, Fletcher JA, Aster JC. BRD-NUT oncoproteins: a family of closely related nuclear proteins that block epithelial differentiation and maintain the growth of carcinoma cells. Oncogene, 27, 2237-2242 (2008).

6) Maruyama T, Farina A, Dey A, Cheong J, Bermudez VP, Tamura T, Sciortino S, Shuman J, Hurwitz J, Ozato K. A Mammalian bromodomain protein, brd4, interacts with replication factor $\mathrm{C}$ and inhibits progression to S phase. Mol. Cell. Biol., 22, 6509-6520 (2002).

7) Alsarraj J, Walker RC, Webster JD, Geiger TR, Crawford NP, Simpson RM, Ozato K, Hunter KW. Deletion of the proline-rich region of the murine metastasis susceptibility gene Brd4 promotes epithelial-to-mesenchymal transition- and stem cell-like conversion. Cancer Res., 71, 3121-3131 (2011).

8) Shimizu Y, Hamazaki Y, Hattori M, Doi K, Terada N, Kobayashi T, Toda Y, Yamasaki T, Inoue T, Kajita Y, Maeno A, Kamba T, Mikami Y, Kamoto T, Yamada T, Kanno T, Yoshikawa K, Ogawa O, Minato N, Nakamura E. SPA-1 controls the invasion and metastasis of human prostate cancer. Cancer Sci., 102, 828-836 (2011).

9) Alsarraj J, Hunter KW. Bromodomain-containing protein 4: a dynamic regulator of breast cancer metastasis through modulation of the extracellular matrix. Int. J. Breast Cancer, 2012, 670632 (2012).

10) Dey A, Nishiyama A, Karpova T, McNally J, Ozato K. Brd4 marks select genes on mitotic chromatin and directs postmitotic transcription. Mol. Biol. Cell, 20, 4899-4909 (2009).

11) Wang R, Li Q, Helfer CM, Jiao J, You J. The bromodomain protein Brd4 associated with acetylated chromatin is important for maintenance of higher-order chromatin structure. J. Biol. Chem., in press (2012).

12) Denis GV, Nikolajczyk BS, Schnitzler GR. An emerging role for bromodomain-containing proteins in chromatin regulation and transcriptional control of adipogenesis. FEBS Lett., 584, 3260-3268 (2010).

13) You J, Croyle JL, Nishimura A, Ozato K, Howley PM. Interaction of the bovine papillomavirus E2 protein with Brd4 tethers the viral DNA to host mitotic chromosomes. Cell, 117, 349-360 (2004).

14) Dey A, Ellenberg J, Farina A, Coleman AE, Maruyama T, Sciortino S, Lippincott-Schwartz J, Ozato K. A bromodomain protein, MCAP, associates with mitotic chromosomes and affects $\mathrm{G}_{2}$-to-M transition. Mol. Cell. Biol., 20, 6537-6549 (2000).

15) Dey A, Chitsaz F, Abbasi A, Misteli T, Ozato K. The double bromodomain protein Brd4 binds to acetylated chromatin during 
interphase and mitosis. Proc. Natl. Acad. Sci. U.S.A., 100, 8758 8763 (2003).

16) Kanno T, Kanno Y, Siegel RM, Jang MK, Lenardo MJ, Ozato K. Selective recognition of acetylated histones by bromodomain proteins visualized in living cells. Mol. Cell, 13, 33-43 (2004).

17) Wu SY, Chiang CM. The double bromodomain-containing chromatin adaptor Brd4 and transcriptional regulation. J. Biol. Chem., 282, 13141-13145 (2007).

18) Paillisson A, Levasseur A, Gouret P, Callebaut I, Bontoux M, Pontarotti P, Monget P. Bromodomain testis-specific protein is expressed in mouse oocyte and evolves faster than its ubiquitously expressed paralogs BRD2, -3, and -4. Genomics, 89, 215-223 (2007).

19) Guo N, Faller DV, Denis GV. Activation-induced nuclear translocation of RING3. J. Cell Sci., 113, 3085-3091 (2000).

20) Wang F, Liu H, Blanton WP, Belkina A, Lebrasseur NK, Denis GV. Brd2 disruption in mice causes severe obesity without Type 2 diabetes. Biochem. J., 425, 71-83 (2010).

21) Garcia-Gutierrez P, Mundi M, Garcia-Dominguez M. Association of bromodomain BET proteins to the chromatin requires dimerization through the conserved motif B. J. Cell Sci., 125, 3671-3680 (2012). 